\title{
A Survey of Patients' Perspectives on Outpatient HIV Care in the Netherlands
}

Esther A. N. Engelhard (1) - Colette Smit · Frank P. Kroon ·

Pythia T. Nieuwkerk - Peter Reiss - Kees Brinkman - Suzanne E. Geerlings

Received: May 18, 2017 / Published online: July 4, 2017

(C) The Author(s) 2017. This article is an open access publication

\section{ABSTRACT}

Introduction: Responding to patients' needs and preferences is important in the delivery of outpatient care. Recent and systematically collected data reflecting human immunodeficiency virus (HIV)-infected patients' opinions on how their outpatient care should be delivered are lacking. Our aim was to identify aspects of care

Enhanced content To view enhanced content for this article go to http://www.medengine.com/Redeem/ 5CD8F060002177A4.

Electronic supplementary material The online version of this article (doi:10.1007/s40121-017-0164-z) contains supplementary material, which is available to authorized users.

E. A. N. Engelhard $(\bowtie) \cdot$ P. Reiss · S. E. Geerlings Division of Infectious Diseases, Department of Internal Medicine, Academic Medical Center of the University of Amsterdam, Amsterdam, The

Netherlands

e-mail: eaengelhard@outlook.com

E. A. N. Engelhard · C. Smit · P. Reiss

Stichting HIV Monitoring, Amsterdam, The

Netherlands

\section{F. P. Kroon}

Department of Infectious Diseases, Leiden

University Medical Center, Leiden, The Netherlands

P. T. Nieuwkerk

Department of Medical Psychology, Academic

Medical Center of the University of Amsterdam,

Amsterdam, The Netherlands that people with HIV in outpatient care in The Netherlands consider important and to evaluate the extent to which the received care meets their expectations.

Methods: We measured patient preferences and experiences in a nationwide sample of HIV-infected patients using a modified, previously validated questionnaire (QUOTE-HIV).

Results: The aspects of care that were considered most important were specific expertise of the care provider in HIV medicine, the care provider taking the patient seriously and receiving adequate information about treatment options. In addition, confidentiality of HIV status at the outpatient clinic was a major concern. Patient experiences were positive, with the majority of the respondents indicating that

P. Reiss

Department of Global Health, Academic Medical Center of the University of Amsterdam, Amsterdam, The Netherlands

P. Reiss

Amsterdam Institute for Global Health and

Development, Amsterdam, The Netherlands

K. Brinkman

Department of Internal Medicine, Onze Lieve

Vrouwe Gasthuis, Amsterdam, The Netherlands 
they always or usually received care in accordance with their preferences.

Conclusion: HIV-infected patients greatly value having care providers with HIV-specific expertise. Safeguarding the privacy of HIV status and the provision of information about treatment options are matters that deserve continuous attention in the delivery of outpatient HIV care.

Keywords: Human immunodeficiency virus; Outpatient care; Patient experiences; Patient preferences; Quality of care

\section{INTRODUCTION}

The importance of responding to patients' needs and preferences (i.e. 'patient-centred care') when delivering health care has been widely recognised [1-3]. In addition to being a goal in itself, evidence suggests that patient-centred care leads to better compliance with medical recommendations and to an improved health status [4]. In HIV-infected patients, patient-centred care has been linked to better adherence to combination antiretroviral therapy (cART) and higher rates of viral suppression [5-7]. However, recent and systematically collected data reflecting HIV-infected patients' opinions on how their outpatient care should be delivered are lacking. Gaining insight into patients' preferences and experience is important, especially given the fact that the number of HIV-infected patients using outpatient care services is growing, owing to their improved survival.

The purpose of this study was to identify aspects of care that are important to HIV-infected patients in outpatient care in The Netherlands and to evaluate the extent to which the received care meets their expectations. To this end, we measured patient preferences and experiences in a national sample of people infected with HIV, using a standardised questionnaire [8].

\section{METHODS}

\section{Setting and Design}

In The Netherlands, HIV outpatient care is provided exclusively in acknowledged HIV treatment centres ( $n=26$ at the time of this study). The care is generally provided and coordinated by specialised clinicians (predominantly infectious disease specialists with experience in HIV medicine) and specialised nurses ("nurse consultants"). All treatment centres collaborate with the collection of data for the ATHENA observational cohort and thus all HIV-infected patients in care (with exception of those who opt out, $2 \%$ ) are registered and monitored in the cohort database [9].

We conducted a cross-sectional study evaluating patients' preferences and experiences in a sample of people with HIV in outpatient care in The Netherlands between July 2013 and December 2014.

\section{Study Population and Procedures}

A nationwide sample of potential participants was selected from the ATHENA database. All HIV-1-infected patients in outpatient care on 25 February 2013, aged 18 or older at time of diagnosis and using cART for at least 6 months, were eligible for participation. We randomly selected samples from each HIV treatment centre in The Netherlands, 1000 patients in total, ensuring a minimum of 20 patients per care facility and accounting for the total number of patients in care in each centre (larger samples in larger treatment centres). We used the pseudonymised study ID number assigned to each HIV patient in care in the cohort to select potential participants.

During their next outpatient visit selected patients were approached for participation by the nurse consultants, who provided them with an information letter and a password for accessing an online survey in Dutch or English. We also offered a paper version of the survey. The Medical Ethics Review Committee of the Academic Medical Centre of the University of Amsterdam exempted this study from written informed consent. We informed all patients about the study and considered consent implicit when a survey was completed online or a hard copy was returned to us.

\section{Patient Characteristics}

Data regarding age, sex, region of origin, socioeconomic status (SES), route of HIV 
transmission, time since initiation of cART and time since HIV diagnosis were extracted from the ATHENA cohort database.

Region of origin, grouped into The Netherlands, sub-Saharan Africa (SSA) and other, was based on the country of birth. We used an area code-based classification system previously described by The Netherlands Institute for Social Research to determine patients' SES [10]. Here, we recoded the five classes as high, middle or low. We grouped route of transmission into men who have sex with men (MSM), heterosexual contact or other/unknown. The last category includes intravenous drug use, as this route of transmission accounts for only a very small proportion of HIV infections in The Netherlands [11].

\section{Outcomes}

The Netherlands Institute for Health Services Research (NIVEL) previously developed a series of disease-specific questionnaires that measure "Quality of Care through the Patient's Eyes" (QUOTE) (in, amongst others, asthma, rheumatic disease and frail elderly) [12]. In QUOTE questionnaires, both patient preferences and experiences are assessed. To measure preferences, patients weigh various care aspects by rating whether statements are "not important", "quite important", "important" or "of greatest importance" (e.g. "The specialist should always explain the advantages and disadvantages of any treatment"). Subsequently, experiences are measured by letting respondents indicate whether they "always", "usually", "sometimes" or "never" receive care as stated (e.g. "My specialist explains the advantages and disadvantages of any treatment").

The HIV-specific version of this questionnaire (QUOTE-HIV) was developed in 1999 using focus groups (patients), tested for its psychometric properties [8] and subsequently tested in a pilot study in the USA [13]. Our aim was to keep adjustments of the instrument to a minimum, but we modified the original QUOTE-HIV questionnaire by removing six statements that we considered no longer applicable (e.g. regarding inpatient care and euthanasia) or difficult to interpret. We also added five statements (concerning the attitude of the care provider, flexibility in care and referral to other disciplines) based on literature review and suggestions of the Dutch Association for people living with HIV. Our final version consisted of 25 statements regarding care provision by the HIV specialist, 21 statements regarding care provision by the nurse consultant, 5 statements regarding general aspects of care and 5 scores (0-100) on specific aspects of care.

\section{Analyses}

We tested for differences in characteristics of respondent and non-respondent characteristics, using $t$ tests, Mann-Whitney $U$ tests and $\chi^{2}$ tests as appropriate.

For each statement, we calculated the proportion of respondents who considered it "important" or "of greatest importance", hereafter jointly referred to as "highly preferred". We then, among the patients who rated the items as "highly preferred", calculated the proportions of patients reporting "always", "usually", "sometimes" or "never" receiving such care.

\section{RESULTS}

Of the 1000 selected potential participants, 958 patients were eligible to respond (i.e. had not died, migrated or switched to another treatment centre recently). A total of 331 respondents from all of the HIV care facilities in The Netherlands completed the questionnaire (response rate: $35 \%$ ). Response rates differed across treatment centres, ranging from 15 to $55 \%$. The proportions of patients originating from The Netherlands (77\%), males (85\%) and men who have sex with men (MSM) (71\%) were significantly higher among respondents than in non-respondents $(50,73,48 \%$ respectively). Respondents were significantly older (median of 51 vs. 47 years), had a significantly higher socioeconomic status (SES) than non-respondents and more often had an undetectable viral load, but were similar in terms of duration of HIV infection and time since cART initiation. 
Table 1 Characteristics of respondents, non-respondents and $p$ values for differences

\begin{tabular}{|c|c|c|c|}
\hline Characteristic & Respondents $(n=331)$ & Non-respondents $(n=627)$ & $p$ value ${ }^{*}$ \\
\hline Age, years, median (IQR) & $51(44-58)$ & $47(40-53)$ & $<0.001$ \\
\hline \multicolumn{4}{|l|}{ Gender } \\
\hline Male & $281(85 \%)$ & $456(73 \%)$ & $<0.001$ \\
\hline \multicolumn{4}{|l|}{ Region of origin } \\
\hline The Netherlands & $255(77 \%)$ & $311(50 \%)$ & \\
\hline sub-Saharan Africa & $28(8 \%)$ & $130(21 \%)$ & \\
\hline Other & $48(15 \%)$ & $186(30 \%)$ & $<0.001$ \\
\hline \multicolumn{4}{|l|}{ Socioeconomic status } \\
\hline High & $91(27 \%)$ & $141(23 \%)$ & \\
\hline Middle & $113(35 \%)$ & $185(30 \%)$ & \\
\hline Low & $119(36 \%)$ & $289(47 \%)$ & $<0.001$ \\
\hline \multicolumn{4}{|l|}{ Transmission risk group } \\
\hline Men who have sex with men & $235(71 \%)$ & $300(48 \%)$ & \\
\hline Heterosexual contact & $77(23 \%)$ & $266(42 \%)$ & \\
\hline Other ${ }^{\mathrm{a}} /$ unknown & $19(6 \%)$ & $61(10 \%)$ & $<0.001$ \\
\hline HIV RNA at participation, $<100$ copies $/ \mathrm{ml}$ & $319(96 \%)$ & $572(91 \%)$ & 0.005 \\
\hline Time since diagnosis, years, median (IQR) & $11(7-16)$ & $10(6-15)$ & 0.24 \\
\hline Time since cART initiation, years, median (IQR) & $9(5-14)$ & $8(5-13)$ & 0.18 \\
\hline
\end{tabular}

${ }^{*}$ Data are presented as no. (\%) unless otherwise specified. $p$ values for the comparison of characteristics between respondents and non-respondents (Fischer's exact test for categorical variables, independent $t$ test for normally distributed continuous variables, Mann-Whitney $U$ test for continuous non-normally distributed variables). Denominators may differ from sample size because of missing values

${ }^{a}$ Injecting drug use, blood (products), needle accident, vertical transmission

The respondents' characteristics are presented in Table 1.

\section{Care Providers: High Preference Issues and Experiences}

Across the entire instrument, the proportion of respondents rating items as "highly preferred" (i.e. "important" or "of greatest importance") ranged from 37 to $98 \%$. Figure 1 presents a selection of the items that were most often rated as "highly preferred", i.e. by $90 \%$ or more of the respondents. The items are grouped by type of care provider and in order of priority. The bars show whether the respondents reported their experience as "always", "usually", "sometimes" or "never" receiving care as stated.

The aspects of care that were most often considered "highly preferred" were having an HIV specialist and nurse consultant with specific expertise in the field of HIV and safeguarding the privacy of HIV status. Other themes that stood out concerned how the care provider treats the patient (taking the patient seriously and a sympathetic/involved attitude) and adequate provision of information regarding treatment (advantages and disadvantages, how to take the medication and possible side effects). 


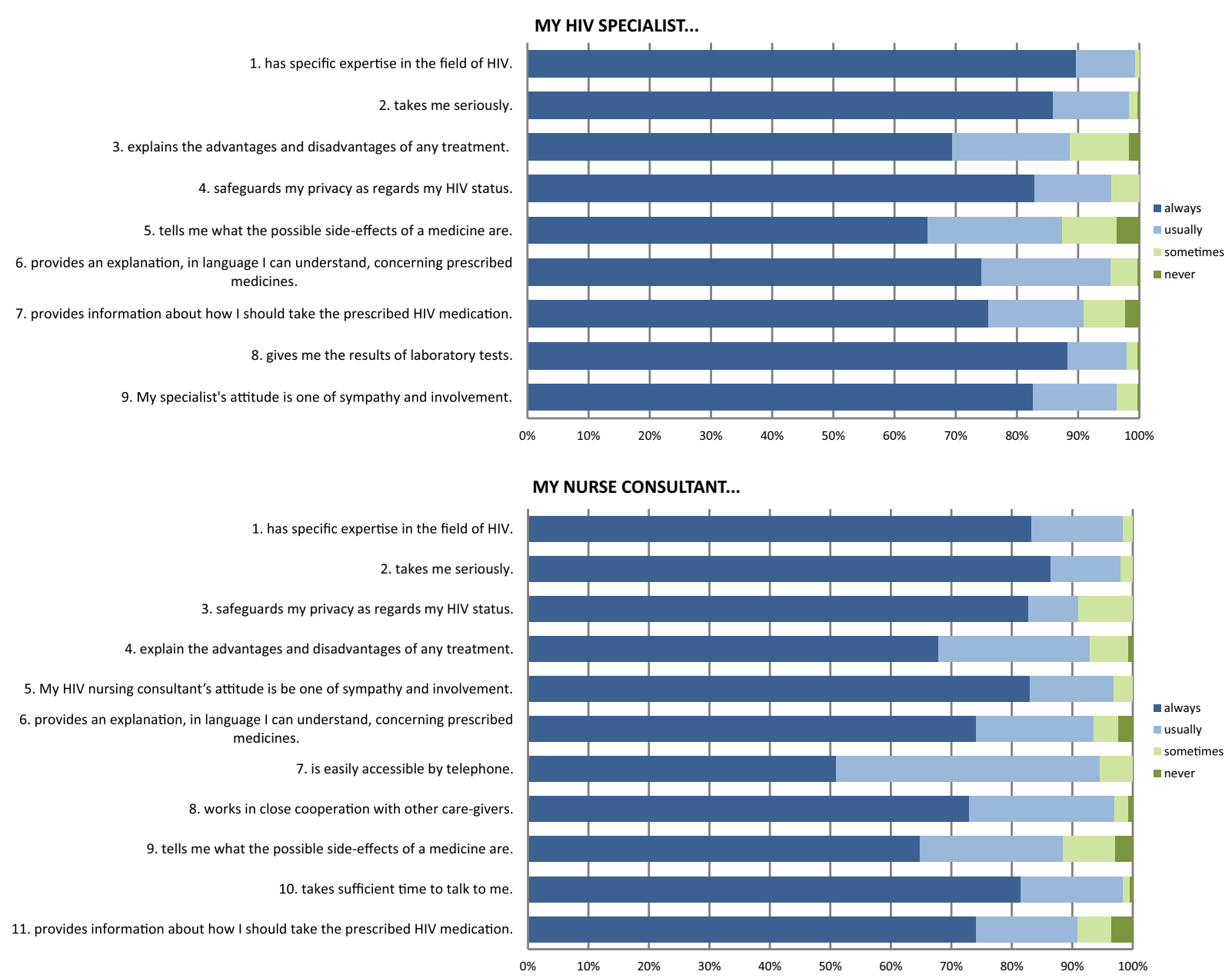

Fig. 1 Care provider aspects of care: experiences of top rated statements. Above statements were rated as "highly preferred" ("important" or "of greatest importance") by $\geq 90 \%$ of respondents and are in order of importance. Bars

Least often viewed as "highly preferred" were aspects regarding waiting times (37\% and 59\% for the medical specialist and nurse consultant respectively), being aware of the patient's situation at home or at work ( $45 \%$ and $62 \%)$, discussing the possibility of requesting a second opinion (56\% and 62\%) and not being disturbed during a consultation with the medical specialist (55\%).

A number of themes were considered important specifically for the nurse consultant. These themes were: being accessible by phone (94\%), working in close cooperation with other care-givers (93\%) and taking sufficient time to talk to the patient (92\%). represent whether these respondents reported their experience as "always", "usually", "sometimes" or "never" receiving such care

Overall, most patients who rated items as "highly preferred" also reported "usually" or "always" getting such care (85-100\%). This applies to all the items in the questionnaire. The rates of reporting "sometimes" or "never" receiving care as stated were somewhat higher in statements regarding the provision of information. Specifically, experiences regarding providing information on advantages and disadvantages of treatments and side effects were somewhat less positive. The median scores that respondents gave to their medical specialists and nurse consultants were 93 and 94 respectively (on a scale of $0-100$ ). 


\section{General Aspects of Outpatient Care}

With regard to priorities in general aspects of care, three themes were covered. The referral to other professionals by HIV care providers was rated as "highly preferred" by $85 \%$ of respondents, who gave a median score of 85 for this item. Being able to schedule an outpatient visit at a suitable time was rated "highly preferred" by $73 \%$, and $92 \%$ of these patients reported usually or always being able to make an appointment at a suitable time. Extensive opening hours of the department for blood draws was considered a "highly preferred" item by $57 \%$ of respondents, and its median score was 82 . Respondents gave the waiting times at the department for blood draws a median score of 76 .

\section{DISCUSSION}

This report provides insight into HIV-infected patients' preferences and experiences in outpatient care in The Netherlands. First, our results show that being treated by care providers with HIV-specific expertise, receiving adequate information about treatment options and the attitude of the care provider (taking the patient seriously and a sympathetic/involved attitude) are considered of greatest importance to people with HIV in outpatient care. Indeed, the three areas 'provider training and competence', 'information and patient education' and provider attitude have consistently been identified as crucial aspects of care delivery in the general $[1,2]$ and HIV-specific $[7,13,14]$ literature on patient-centred care. Statements concerning accessibility (flexibility in care and waiting times) were considered important, but evidently of lower priority in our study population than the earlier mentioned items. Results from previous studies suggest that priorities may vary per patient population. For instance, in a study in patients with five different conditions, de Boer et al. found that the top ten priorities in patients with diabetes were evenly distributed among provider attitude and information, while rheumatoid arthritis patients prioritised items regarding accessibility of care (rapid referral and availability of provider when discomfort increases) [15].

Second, our results highlight the fact that the confidentiality of HIV status at the outpatient clinic is a major concern for patients. We have no data to verify whether this issue is specific for HIV. However, given the fact that the stigma surrounding HIV continues to be a widespread problem [16], it is highly probable that HIV-positive patients are particularly concerned about how their care providers handle information regarding their HIV status.

Third, our results suggest that overall, delivery of outpatient HIV care in The Netherlands meets patients' expectations. There is some room for improvement with respect to waiting times at the department for blood draws and provision of information regarding treatment (in particular, advantages and disadvantages, how to take the medication and possible side effects).

\section{Strengths and Limitations}

The strength of this study is that we have data from a national sample of HIV-infected patients in which each of the designated HIV care facilities in the country is represented. In addition, we used an HIV-specific instrument that was developed together with patients and that measures preferences as well as experiences. Furthermore, to reduce potential sampling bias related to online survey research [17], respondents were provided with the option of filling out a hard copy version of the questionnaire.

There are however also important limitations to take into account. Despite our efforts to recruit a nationally representative sample of HIV-infected patients, people from The Netherlands, MSM and patients with a higher SES were overrepresented in the respondents. Moreover, the response rate in this study was relatively low $(35 \%)[12,18]$, with rates differing considerably across the centres. There is a possibility that patients' experiences impacted their willingness to respond. Indeed, previous studies have found an association between more favourable patient perceptions and higher participation rates in health survey studies [18]. The fact that respondents were more likely to 
have an undetectable viral load supports this notion. However, evidence also suggests that the overall impact of non-response bias may be small and that increasing participation may not change conclusions of patient perception surveys $[19,20]$. In addition, our adjustment for SES has its limitations because it is based on area codes and not on individual patient data (e.g. education and occupational status). In the absence of a validated instrument applicable to the current era of HIV care, we used an instrument that was developed more than a decade ago and has not been used extensively. Furthermore, since the development of this instrument much progress has been made in terms of methods for measuring important aspects of the patient care experience. However, in view of the fact that our instrument was developed systematically and jointly with patients and covers HIV-specific themes that are still relevant, we believe our results have yielded useful information.

\section{Implications}

Our results underline the importance of providing information that meets the needs and expectations of patients. Receiving adequate information has emerged as a particular area of concern in many previous studies [21-24]. Our results therefore confirm that those involved with the delivery of care should continuously be engaged with which information patients wish to receive, how this information should be provided and whether the provision indeed meets patients' needs.

In addition, our data show that confidentiality of patients' HIV status is of great importance in clinical practice. Practices should consider assessing patients' specific concerns so that measures to minimise confidentiality breaches can be identified. Examples of such issues include the physical environment (e.g. separating the reception area from the waiting area), staff-to-staff contact and the manner of 'calling the patient into consultation'.

Respondents reported positive experiences across all aspects of care covered by the questionnaire. Given that the HIV care system in The
Netherlands has a long history of being assigned to providers and facilities with HIV-related experience, these positive results may reflect an overall good quality of HIV care. However, considering that the questionnaire was developed in the beginning of the cART era, the questionnaire may have failed to capture a number of themes that are important to the current HIV-infected population [4]. Acknowledged areas of particular interest may include psychosocial support $[25,26]$, patients' involvement in medical decisions (i.e. 'shared decision making') [1,7] and peer support [26]. Other topics that are worth investigating are patients' opinions on relocation of services (including the management of non-HIV co-morbidities) from specialised centres to peripheral health facilities or general practitioners and patients' wishes regarding the frequency and duration of appointments. In the absence of a gold standard, the HIV care field would benefit from a novel and validated instrument to assess HIV-infected patients' preferences and experiences. Crucial for the development of such an instrument is that patients are involved throughout the process [27]. In addition, the development process should be guided by an established framework. One of the 'patient-centred care' frameworks that has been used extensively in research is the Institute of Medicine's six dimensions of patient-centred care $[1,18]$. The dimensions consist of: (1) respect for patients' values, preferences and expressed needs; (2) information, communication and education; (3) coordination and integration of care; (4) emotional support-relieving fear and anxiety; (5) physical comfort; (6) involvement of family and friends [28].

\section{CONCLUSION}

The data in this study reflect HIV-infected patients' opinions on outpatient care. Care provider expertise and attitude, information and confidentiality of HIV status stand out as important aspects of care. Experiences were generally positive regarding the themes that were addressed, but there is a need to develop a new validated instrument that is applicable to the current era of HIV care. 


\section{ACKNOWLEDGEMENTS}

This work was supported by Aids Fonds, research grant no. 2011015. The ATHENA cohort is maintained by the Stichting HIV Monitoring and supported by a grant from The Netherlands Ministry of Health, Welfare and Sport. We would like to thank all participants for their contributions and the physicians and nurse consultants for making the collection of the data possible. Data of the ATHENA cohort, maintained by the Stichting HIV Monitoring, were used in this study. No funding or sponsorship was received for this study or publication of this article.

EANE, FPK, KB, CS and SEG contributed to the design of the study. EANE collected/analysed the data and prepared the manuscript. CS, SEG and $\mathrm{KB}$ supervised the development of work. All named authors meet the International Committee of Medical Journal Editors (ICMJE) criteria for authorship for this manuscript, take responsibility for the integrity of the work as a whole and have given final approval for the version to be published.

Disclosures. Suzanne E. Geerlings has served on an advisory board for Gilead Sciences. Kees Brinkman serves on advisory boards for MSD, Gilead, BMS, Viiv and Janssen, for which he has received remuneration. Peter Reiss has received independent scientific grant support from Gilead Sciences, Janssen Pharmaceuticals Inc., Merck \& Co., BristolMyers Squibb and ViiV Healthcare through his institution. He has served on a scientific advisory board for Gilead Sciences and a data safety monitoring committee for Janssen Pharmaceuticals Inc., and he has chaired a scientific symposium by ViiV Healthcare for which his institution received remuneration. Esther A. N. Engelhard, Colette Smit, Frank P. Kroon and Pythia T. Nieuwkerk have nothing to disclose.

Compliance with Ethics Guidelines. The Medical Ethics Review Committee of the Academic Medical Centre of the University of Amsterdam exempted this study from written informed consent. We informed all patients about the study and considered consent implicit when a survey was returned to us. We did not include patients who had opted out of registration in the ATHENA cohort.

Data Availability. The data sets generated during and/or analysed during the current study are available from the corresponding author on reasonable request.

Open Access. This article is distributed under the terms of the Creative Commons Attribution-NonCommercial 4.0 International License (http://creativecommons.org/licenses/ by-nc/4.0/), which permits any noncommercial use, distribution, and reproduction in any medium, provided you give appropriate credit to the original author(s) and the source, provide a link to the Creative Commons license, and indicate if changes were made.

\section{REFERENCES}

1. Kitson A, Marshall A, Bassett K, Zeitz K. What are the core elements of patient-centred care? A narrative review and synthesis of the literature from health policy, medicine and nursing. J Adv Nurs. 2013;69(1):4-15. doi:10.1111/j.1365-2648.2012. 06064.x.

2. Cheraghi-Sohi S, Bower P, Mead N, McDonald R, Whalley D, Roland M. What are the key attributes of primary care for patients? Building a conceptual 'map' of patient preferences. Health Expect: Int J Public Particip Health Care Health Policy. 2006;9(3):275-84. doi:10.1111/j.1369-7625.2006. 00395.x.

3. Groene O. Patient centredness and quality improvement efforts in hospitals: rationale, measurement, implementation. Int J Qual Health Care: $\mathrm{J}$ Int Soc Qual Health Care/ISQua. 2011;23(5):531-7. doi:10.1093/intqhc/mzr058.

4. Browne K, Roseman D, Shaller D, Edgman-Levitan S. Analysis and commentary. Measuring patient experience as a strategy for improving primary care. Health Aff. 2010;29(5):921-5. doi:10.1377/hlthaff. 2010.0238 .

5. Beach MC, Keruly J, Moore RD. Is the quality of the patient-provider relationship associated with better adherence and health outcomes for patients with HIV? J Gen Intern Med. 2006;21(6):661-5. doi:10. 1111/j.1525-1497.2006.00399.x. 
6. Schneider J, Kaplan SH, Greenfield S, Li W, Wilson IB. Better physician-patient relationships are associated with higher reported adherence to antiretroviral therapy in patients with HIV infection. J Gen Intern Med. 2004;19(11):1096-103. doi:10.1111/j.1525-1497.2004.30418.x.

7. Land L, Nixon S, Ross JD. Patient-derived outcome measures for HIV services in the developed world: a systematic review. Int J STD AIDS. 2011;22(8):430-5. doi:10.1258/ijsa.2011.010450.

8. Hekkink CF, Sixma HJ, Wigersma L, Yzermans CJ, Van Der Meer JT, Bindels PJ, et al. QUOTE-HIV: an instrument for assessing quality of HIV care from the patients' perspective. QualSaf Health Care. 2003;12(3):188-93.

9. Stichting HIV Monitoring. Annual report 2015. https://www.hiv-monitoring.nl/files/1514/6424/ 7604/Stichting_HIV_Monitoring_annual_report_ 2015.pdf. Accessed 23 Dec 2016.

10. The Netherlands institute for social research. Neighbourhood status development in the Netherlands 1998-2010, summary. 2012. https://www.scp.nl/ english/Publications/Summaries_by_year/Summaries_ 2012/Neighbourhood_status_development_in_the Netherlands_1998_2010. Accessed 14 Apr 2015.

11. van Sighem A, Boender TS, Wit FWNM, Smit C, Matser A, Reiss P. Human immunodeficiency virus (HIV) infection in the Netherlands. Monitoring report 2016. https://www.hiv-monitoring.nl/files/ 6614/7999/7485/HIV_Monitoring_Report_2016_ 24_Nov.pdf. Accessed 23 Dec 2016

12. van der Eijk I, Sixma H, Smeets T, Veloso FT, Odes S, Montague S, et al. Quality of health care in inflammatory bowel disease: development of a reliable questionnaire (QUOTE-IBD) and first results. Am J Gastroenterol. 2001;96(12):3329-36. doi:10.1111/j.1572-0241.2001.05334.x.

13. Moore BR, Simpson K, Kaye W, Swanson KA. Piloting a method to measure satisfaction with HIV care. Am J Med Qual. 2010;25(5):384-91. doi:10. $1177 / 1062860610371974$.

14. Hekkink CF, Wigersma L, Yzermans CJ, Bindels PJ. HIV nursing consultants: patients' preferences and experiences about the quality of care. J Clin Nurs. 2005;14(3):327-33. doi:10.1111/j.1365-2702.2004. 01061.x.

15. de Boer D, Delnoij D, Rademakers J. Do patient experiences on priority aspects of health care predict their global rating of quality of care? A study in five patient groups. Health expect: Int J Public Particip In Health Care Health Policy. 2010;13(3):285-97. doi:10.1111/j.1369-7625.2010.00591.x.
16. Earnshaw VA, Lang SM, Lippitt M, Jin H, Chaudoir SR. HIV stigma and physical health symptoms: do social support, adaptive coping, and/or identity centrality act as resilience resources? AIDS Behav. 2015;19(1):41-9. doi:10.1007/s10461-014-0758-3.

17. Jenkins PJ, Sng S, Brooksbank K, Brooksbank AJ. Socioeconomic deprivation and age are barriers to the online collection of patient reported outcome measures in orthopaedic patients. Ann R Coll Surg Engl. 2016;98(1):40-4. doi:10.1308/rcsann.2016.0007.

18. Castle NG, Brown J, Hepner KA, Hays RD. Review of the literature on survey instruments used to collect data on hospital patients' perceptions of care. Health Serv Res. 2005;40(6 Pt 2):1996-2017. doi:10. 1111/j.1475-6773.2005.00475.x.

19. Lasek RJ, Barkley W, Harper DL, Rosenthal GE. An evaluation of the impact of nonresponse bias on patient satisfaction surveys. Med Care. 1997;35(6):646-52.

20. Perneger TV, Chamot E, Bovier PA. Nonresponse bias in a survey of patient perceptions of hospital care. Med Care. 2005;43(4):374-80.

21. Groene $\mathrm{O}$, Lombarts $\mathrm{MJ}$, Klazinga $\mathrm{N}$, Alonso J, Thompson A, Sunol R. Is patient-centredness in European hospitals related to existing quality improvement strategies? Analysis of a cross-sectional survey (MARQuIS study). Qual Saf Health Care. 2009;18(Suppl 1):i44-50. doi:10.1136/qshc. 2008.029397.

22. Dwamena F, Holmes-Rovner M, Gaulden CM, Jorgenson S, Sadigh G, Sikorskii A, et al. Interventions for providers to promote a patient-centred approach in clinical consultations. Cochrane Database Syst Rev. 2012;12:Cd003267. doi:10.1002/ 14651858.CD003267.pub2.

23. Kleefstra SM, Zandbelt LC, de Haes HJ, Kool RB. Trends in patient satisfaction in Dutch university medical centers: room for improvement for all. BMC Health Serv Res. 2015;15:112. doi:10.1186/ s12913-015-0766-7.

24. Jacobi CE, Boshuizen HC, Rupp I, Dinant HJ, van den Bos GA. Quality of rheumatoid arthritis care: the patient's perspective. Int J Qual Health Care: J Int Soc Qual Health Care/ISQua. 2004;16(1):73-81. doi:10.1093/intqhe/mzh009.

25. Davis-Michaud M, Yurk R, Lansky D, Asch S, Wu AW. Quality care for people with HIV/AIDS: patients' perspectives. HIVClin Trials. 2004;5(6):406-15.

26. Minick SG, Stafford CL, Kertz BL, Cully JA, Stanley MA, Davila JA, et al. Veterans' perspectives on 
interventions to improve retention in HIV care. PLoS One. 2016;11(2):e0148163. doi:10.1371/ journal.pone.0148163.

27. Wiering B, de Boer D, Delnoij D. Patient involvement in the development of patient-reported outcome measures: a scoping review. Health expect: Int J Public Particip Health Care Health Policy. 2016. doi:10.1111/hex.12442.
28. van Leijen-Zeelenberg JE, Huismans GW, Bisschop JA, Brunings JW, van Raak AJ, Ruwaard D, et al. Experiences and preferences of patients visiting an otorhinolaryngology outpatient clinic: a qualitative study. Health Expect: Int J Public Particip Health Care Health Policy. 2015. doi:10.1111/hex.12347. 